\title{
Ovário-histerectomia: estudo experimental comparativo entre as abordagens laparoscópica e aberta na espécie canina. II- Evolução clínica pós-operatória
}

\author{
[Ovariohysterectomy: experimental and comparative study between laparoscopic and conventional approaches. II- \\ Post-operative clinical evolution] \\ C. Malm ${ }^{1}$, P.R. Savassi-Rocha ${ }^{2}$, V.A. Gheller ${ }^{1}$, H.P. Oliveira ${ }^{1}$, A.R. Lamounier ${ }^{3}$, V. Foltynek ${ }^{4}$ \\ ${ }^{1}$ Escola de Veterinária da UFMG \\ Caixa Postal 567 \\ 30123-970 - Belo Horizonte, MG \\ ${ }^{2}$ Instituto Alfa de Gastroenterologia - Hospital das Clínicas da UFMG \\ ${ }^{3}$ Escola de Veterinária - PUC - Betim \\ ${ }^{4}$ Estudante de Mestrado - Escola de Veterinária da UFMG
}

\begin{abstract}
RESUMO
Avaliou-se a evolução clínica pós-operatória de 30 cadelas sem raça definida, durante sete dias, aleatoriamente distribuídas em dois grupos de 15 animais, submetidas à ovário-histerectomia $(\mathrm{OVH})$ pelas abordagens laparoscópica (grupo I) e aberta (grupo II). Avaliaram-se os parâmetros de comportamento, fisiológicos e de complicações na ferida cirúrgica. Foi utilizada uma escala descritiva para avaliação da dor e das complicações pós-operatórias. Não foram encontradas diferenças significativas entre os grupos quanto às variáveis: locomoção, postura, interferência na ferida cirúrgica, tensão abdominal, vocalização, apetite, evacuação, freqüências cardíaca e respiratória e temperatura corporal. Quando as variáveis de comportamento e fisiológicas foram avaliadas em conjunto (escore 1), observou-se maior dor pós-operatória apenas no segundo dia do pós-operatório nas cadelas submetidas à cirurgia aberta. Quando as complicações das feridas cirúrgicas foram avaliadas em conjunto (escore 2), observou-se maior ocorrência dessas nos animais do grupo 1 . O escore total (somatória dos escores 1 e 2) mostrou que a recuperação pós-operatória foi semelhante nas duas abordagens estudadas.
\end{abstract}

Palavras-chave: cadela, laparoscopia, ovário-histerectomia, dor pós-operatória

\begin{abstract}
It was compared the post-operative phase (seven days) of 30 bitches, randomly divided in two groups of 15 animals and submitted to laparoscopic (group I) and conventional ovariohisterectomy (group II). Both groups were compared by behavioral and physiological parameters, as well as post-operative complications of the surgical site. A descriptive scale was used to evaluate the behavioral and post operative complications. No statistical differences were observed between the groups when the locomotion, posture, surgical wound interference, abdominal tension, vocalization, appetite, cardiac and respiratory rates, body temperature and evacuation were individually analyzed. When the behavioral and physiological parameters were evaluated together (score 1), there was evidence of major post-operative pain only in the second day in bitches submitted to conventional surgery. When surgical wound complications were analyzed as a whole (score 2), they were more frequent in group I. The total score (score $1+$ score 2) showed similarity to post-operative recovery between the two surgical approaches.
\end{abstract}

Keywords: bitch, laparoscopy, ovariohysterectomy, post-operative pain

Recebido para publicação em 13 de julho de 2004

Recebido para publicação, após modificações, em 25 de maio de 2005

E-mail: malm@vet.ufmg.br 


\section{INTRODUÇÃO}

Na medicina humana, estudos comprovam que a cirurgia laparoscópica oferece vantagens quando comparada com a aberta. Pequenas incisões, menor trauma tissular, melhor função respiratória, menos dor pós-operatória, menor tempo de hospitalização e recuperação póscirúrgica mais rápida são citados por Graves et al. (1991), Soper e Dunnegan (1993) e SavassiRocha (1995). A via laparoscópica é muito atraente, pois leva à diminuição dos custos hospitalares e do tempo de hospitalização, além de promover retorno mais rápido do paciente às suas atividades normais. Numerosas pesquisas têm demonstrado as vantagens e as possíveis complicações dessa abordagem (Andreollo et al., 1999; Hendolin et al., 2000). Considerando a qualidade da recuperação pós-operatória do paciente humano, o grau de dor nessa fase tem sido investigado e correlacionado com complicações pulmonares, estados mórbidos, estresse e tempo de recuperação (Frazee et al., 1991; Hendolin et al., 2000).

Na medicina veterinária, a laparoscopia é uma abordagem inovadora que tem sido utilizada em vários procedimentos cirúrgicos, incluindo a ovário-histerectomia $(\mathrm{OVH})$ (Minami et al., 1997; Aguilar et al., 1997; Brun, 1999; Malm et al., 2004). No entanto, não foram citados na literatura consultada estudos comparativos entre as abordagens laparoscópica e aberta. $\mathrm{Na}$ introdução dessa nova abordagem cirúrgica, é pertinente o estudo da evolução pós-operatória em animais, considerando que, em humanos, ela oferece muitas vantagens. Quanto à dor pósoperatória e à recuperação do paciente animal, existe grande interesse nesse tipo de pesquisa, uma vez que, atualmente, a preocupação ética com o sofrimento e o bem-estar animal é uma questão certa e indiscutível (Rollin, 2002).

Este trabalho teve como objetivo avaliar a evolução clínica pós-operatória (parâmetros de comportamento, fisiológicos e complicações na ferida cirúrgica), durante sete dias, em cadelas submetidas à ovário-histerectomia pelas abordagens laparoscópica e aberta.

\section{MATERIAL E MÉTODOS}

Foram utilizadas 30 cadelas, hígidas, sem raça definida, jovens (oito meses a dois anos de idade) pesando entre 6,5 e $19,0 \mathrm{~kg}$. Os animais permaneceram, durante 15 dias, em canis com solário para fins de observação e adaptação, sendo alimentados com ração comercial ${ }^{1}$. Em seguida, foram alojados em canis individuais e distribuídos ao acaso formando dois grupos de 15 .

No pré-operatório imediato, procedeu-se à mensuração das freqüências cardíaca e respiratória e da temperatura corporal, bem como à administração de cefalotina sódica ${ }^{2}$ (30mg/kg/IV), 30 minutos antes da intervenção cirúrgica. $\mathrm{O}$ preparo do equipamento laparoscópico para os procedimentos cirúrgicos, esterilização do material e montagem da equipe cirúrgica foram descritos por Malm et al. (2004). Os animais foram submetidos aos procedimentos pré-anestésico e anestésico e, em seguida, à $\mathrm{OVH}$ laparoscópica (grupo I) e OVH aberta (grupo II), segundo descrição feita por Malm et al. (2004). Logo após o término do procedimento cirúrgico, procedeu-se ao curativo das feridas cirúrgicas com gaze estéril e esparadrapo e colocação do colar elisabetano. Não foram administradas drogas analgésicas no pósoperatório, para que não houvesse qualquer interferência nas avaliações clínicas e comportamentais propostas. Os animais permaneceram em canis individuais durante $\mathrm{o}$ retorno anestésico.

Os exames clínicos, para avaliação pósoperatória, iniciaram-se na manhã seguinte, e foram realizados, uma vez ao dia, entre 8 e 12 horas, durante sete dias, com a participação de três avaliadores. As interpretações foram registradas em fichas de evolução clínica pósoperatória (Tab. 1). Os curativos e o colar elisabetano eram removidos para permitir melhores avaliações clínicas e comportamentais.

\footnotetext{
${ }^{1}$ Herói - GUABI - Mogiana Alimentos S.A, Campinas, Brasil.

${ }^{2}$ Cefalotina - ARISTON - Indústria Química e Farmacêutica Ltda., São Paulo, Brasil.
} 
Tabela 1. Ficha de evolução clínica pós-operatória com escala descritiva de avaliação de dor em cadelas submetidas à ovário-histerectomia laparoscópica e aberta

\begin{tabular}{|c|c|c|c|}
\hline & Data: & & Abordagem cirúrgica: \\
\hline & Parâmetro & Escore & Descrição \\
\hline & Comportamental & & \\
\hline \multirow{21}{*}{$\begin{array}{l}\ddot{\overline{0}} \\
\ddot{0} \\
\bar{c}\end{array}$} & 1 Locomoção espontânea & $\begin{array}{l}0 \\
1\end{array}$ & $\begin{array}{l}\text { Sim } \\
\text { Não }\end{array}$ \\
\hline & 2 Postura corporal & $\begin{array}{l}0 \\
1 \\
2 \\
3\end{array}$ & $\begin{array}{l}\text { Em pé } \\
\text { Deitado e levanta ao estímulo } \\
\text { Deitado, levanta ao estímulo e cifose } \\
\text { Deitado e não levanta ao estímulo }\end{array}$ \\
\hline & 3 Interferência do animal na ferida cirúrgica & $\begin{array}{l}0 \\
1\end{array}$ & $\begin{array}{l}\text { Não } \\
\text { Sim }\end{array}$ \\
\hline & 4 Reação do animal à palpação da área operada & $\begin{array}{l}0 \\
1 \\
2 \\
3\end{array}$ & $\begin{array}{l}\text { Sem reação } \\
\text { Leve desconforto, atento à palpação, olhar para a área manipulada. } \\
\text { Desconforto, movimentação, agitação, tentativas para sair do } \\
\text { estímulo da palpação } \\
\text { Reação agressiva, movimentação, vocalização, retirada do } \\
\text { estímulo, tentativa de morder }\end{array}$ \\
\hline & 5 Abdômen & $\begin{array}{l}0 \\
1\end{array}$ & $\begin{array}{l}\text { Normal } \\
\text { Tensão da parede abdominal }\end{array}$ \\
\hline & 6 Vocalização & $\begin{array}{l}0 \\
1\end{array}$ & $\begin{array}{l}\text { Não } \\
\text { Sim }\end{array}$ \\
\hline & & 0 & Normorexia \\
\hline & 7 Apetite & 1 & Hiporexia \\
\hline & & 2 & Anorexia \\
\hline & Fisiológico & & \\
\hline & & 0 & $0 \%$ a $15 \%$ acima do valor no pré-operatório \\
\hline & 8 Freä̈ência cardíaca & 1 & De $16 \%$ a $29 \%$ acima do valor no pré-operatório \\
\hline & 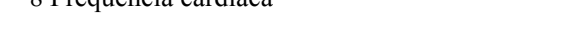 & 2 & De $30 \%$ a $45 \%$ acima do valor no pré-operatório \\
\hline & & 3 & Acima de $45 \%$ do valor no pré-operatório \\
\hline & & 0 & $0 \%$ a $15 \%$ acima do valor no pré-operatório \\
\hline & 9 Freaïência resniratória & 1 & De $16 \%$ a $29 \%$ acima do valor no pré-operatório \\
\hline & у гіеquencta respratona & 2 & De $30 \%$ a $45 \%$ acima do valor no pré-operatório \\
\hline & & 3 & Acima de $45 \%$ do valor no pré-operatório \\
\hline & 10 Tempernture cornoral & 0 & Temperatura normal \\
\hline & 10 i emperatura corporal & 1 & Temperatura retal acima da variação normal para a espécie canina \\
\hline & 11 Evacuação & $\begin{array}{l}0 \\
1\end{array}$ & $\begin{array}{l}\text { Sim } \\
\text { Não }\end{array}$ \\
\hline \multirow{13}{*}{ 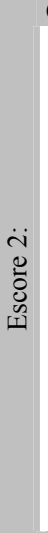 } & Complicação pós-operatória relacionada ao sítio ci & úrgico & \\
\hline & & 0 & Ausente \\
\hline & 12 Enfisema subcutâne & 1 & Discreto \\
\hline & 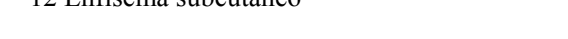 & 2 & Moderado \\
\hline & & 3 & Acentuado \\
\hline & 13 Hematoma & 0 & Não \\
\hline & & 1 & Sim \\
\hline & 14 Seroma & 0 & Não \\
\hline & & & \\
\hline & 15 Infecção & 0 & Não \\
\hline & & 1 & Sim \\
\hline & 16 Deiscência & 0 & Não \\
\hline & & 1 & Sim \\
\hline
\end{tabular}


Para avaliar a dor e o desconforto no período pós-operatório, foi utilizada uma ficha de evolução clínica pós-operatória com uma escala descritiva que permitiu a análise de sete parâmetros de comportamento e quatro parâmetros fisiológicos (Tab. 1). Cada parâmetro contém as descrições de diferentes manifestações exibidas pelo animal durante o exame clínico. Para cada parâmetro e suas respectivas manifestações, foram atribuídos valores numéricos (escores) (Tab. 1). No momento da avaliação, os observadores atribuíam um valor a cada manifestação exibida pelo animal. Os valores obtidos foram posteriormente somados para determinação do escore 1 , que mostrou a evolução da dor e as alterações dos parâmetros fisiológicos, em cada animal, no período pósoperatório. A contagem variou de zero a 19 pontos, sendo que o valor zero indicou a ausência de dor e de alterações nos parâmetros fisiológicos, e o valor 19 indicou o grau máximo de resposta para cada variável citada. Essa ficha foi também utilizada para registrar a ocorrência e a evolução de complicações nas feridas cirúrgicas, por animal, nas duas abordagens, mediante atribuição de valores numéricos (escores), cuja soma representou o escore 2 (Tab. 1). A contagem variou de zero a sete pontos, em que $o$ valor zero indicou ausência de complicações e o sete, a ocorrência de todas as complicações estudadas.

O escore 1 médio foi obtido pela somatória dos escores 1 de todas as 15 cadelas, em cada dia do pós-operatório, para cada abordagem, dividido pelo número de animais estudados em cada grupo $(n=15)$. Idêntico procedimento foi feito para a obtenção de escore 2 médio.

O escore total (escore $1+$ escore 2 ) representou a somatória das alterações nos parâmetros comportamental, físiológico e complicação da ferida cirúrgica. Mostrou a evolução clínica pósoperatória e a qualidade da recuperação dos pacientes nesse período. Nesse caso, os valores numéricos variaram de zero a 26 pontos, de modo que o valor zero indicou ausência de dor e de complicações e o valor 26, o grau máximo de dor com ocorrência de todas as complicações.

Durante sete dias de pós-operatório, foram realizadas 105 avaliações em cada grupo. Os dados obtidos no pré-cirúrgico, antes de qualquer manipulação dos animais (avaliação zero), serviram como controle para as demais avaliações no pós-operatório.

O estudo estatístico constou de: análise de variância com médias comparadas pelos testes Kruskall-Wallis ou Wilcoxon para as variáveis não paramétricas (palpação do sítio cirúrgico, tensão abdominal, apetite, vocalização e enfisema subcutâneo); análise de variância com médias comparadas pelo teste Duncan para os dados numéricos (freqüências cardíaca e respiratória e temperatura corporal) e dispersão de freqüência $\left(\chi^{2}\right)$ utilizando-se o teste exato de Fisher para as variáveis qualitativas com respostas dicotômicas como locomoção, postura, evacuação, interferência nas feridas cirúrgicas, hematoma, seroma, infecção e deiscência. Os escores 1,2 e total foram submetidos aos testes Kruskall-Wallis, para comparação ao longo dos sete dias do pós-operatório no mesmo grupo, e Wilcoxon, para comparação entre os dois grupos ao longo do período pós-operatório. Diferenças foram consideradas significativas quando $\mathrm{P}<0,05$.

\section{RESULTADOS}

Não houve diferença entre as duas abordagens cirúrgicas estudadas quanto à postura corporal (Fig. 1), interferência na ferida cirúrgica (Fig. 2), tensão abdominal (Fig. 3), vocalização (Fig. 4), apetite (Fig.5), freqüências cardíaca e respiratória, temperatura corporal e evacuação. Para todos os animais, a locomoção espontânea normal ocorreu a partir do primeiro dia do pós-operatório.

Com relação às reações à palpação do sítio cirúrgico, nas cirurgias laparoscópicas (grupo I), houve diferença $(\mathrm{P}<0,05)$ quando o primeiro dia de pós-operatório foi comparado com os quatro últimos dias. Maior número de animais apresentou mais desconforto e dor à palpação da área operada no primeiro dia em comparação aos dias 4, 5, 6 e 7, ocorrendo diminuição na sensação dolorosa na evolução do pós-operatório (Fig. 6). Nas demais comparações entre os dias, não foram observadas diferenças $(\mathrm{P}>0,05)$. No grupo das cirurgias abertas, observou-se diferença $(\mathrm{P}<0,05)$ dos dias 1,2 e 3 quando comparados aos dias 5, 6 e 7. Nesses últimos, houve apenas evidência de leve desconforto durante a palpação da área operada (Fig. 7). Nenhuma diferença $(\mathrm{P}>0,05)$ foi observada no dia 4 em relação aos demais dias. 


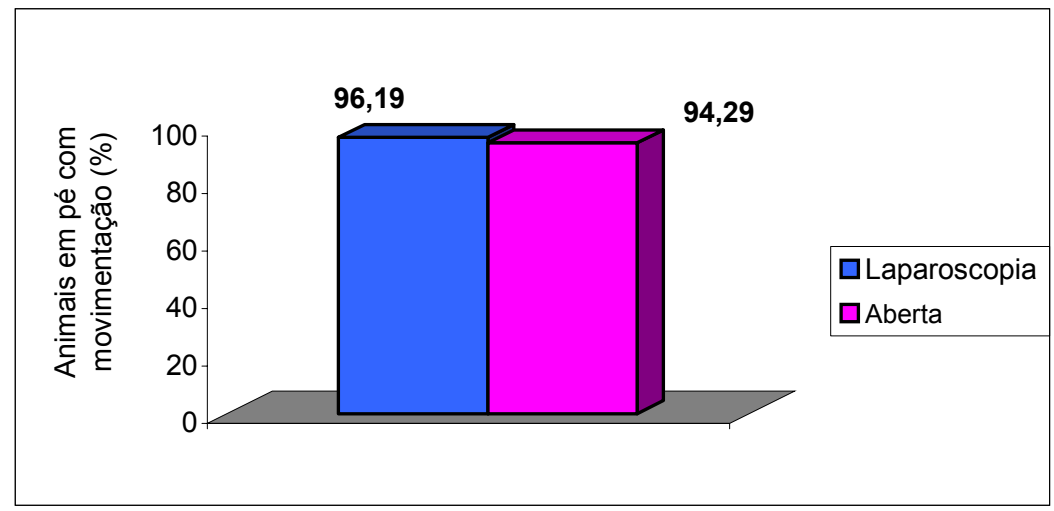

Figura 1. Percentual de observações para animais em pé com movimentação espontânea (escore 0) durante o pós-operatório de sete dias $(n=210)$, de acordo o grupo estudado $(\mathrm{P}=0,834$ - teste exato de Fisher).

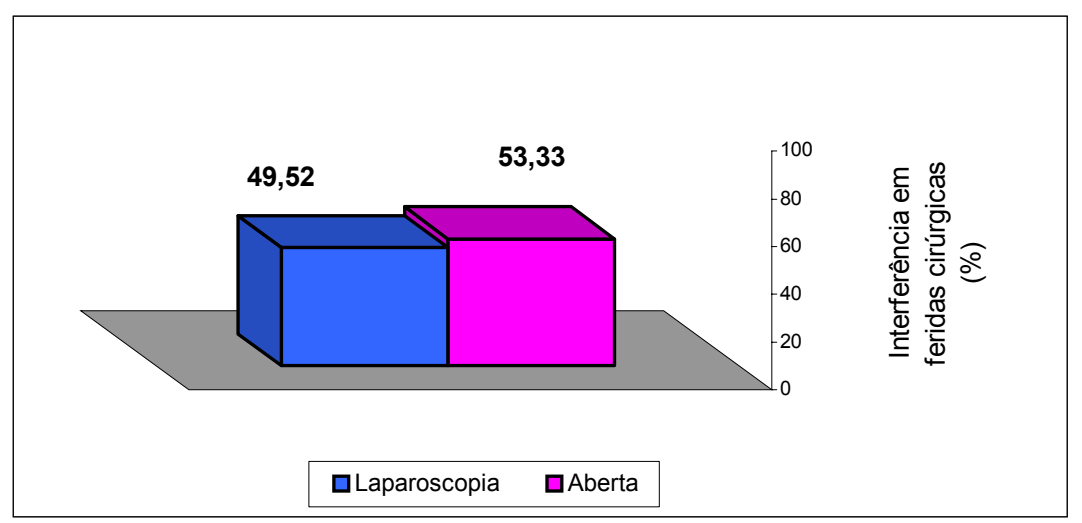

Figura 2. Percentual de observações de interferência na ferida cirúrgica durante o pós-operatório de sete dias $(n=210)$, de acordo com o grupo estudado $(P=0,755$ - teste exato de Fisher).

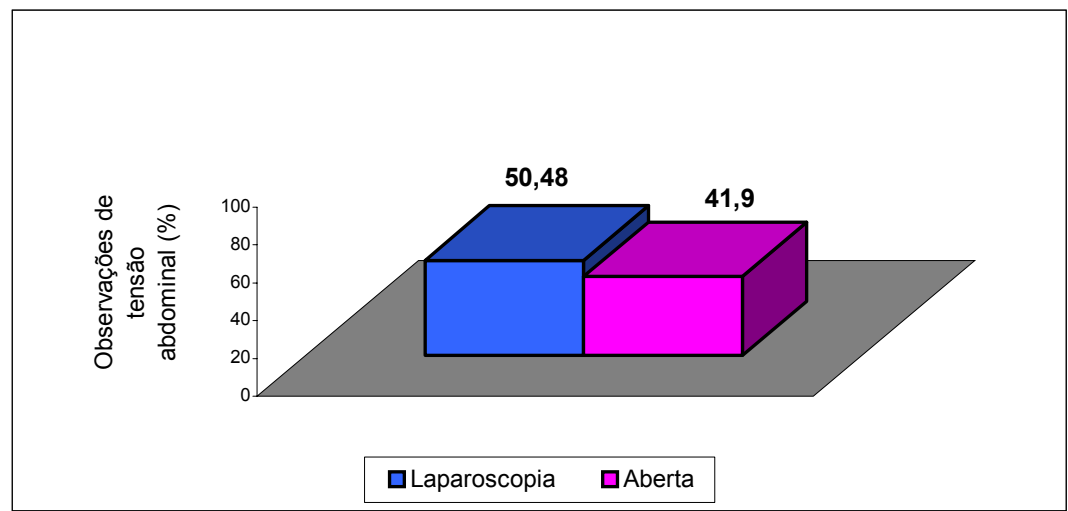

Figura 3. Percentual de observações de tensão abdominal pós-operatória em 210 observações, nos dois grupos estudados $(\mathrm{P}=0,134$ - teste exato de Fisher $)$. 


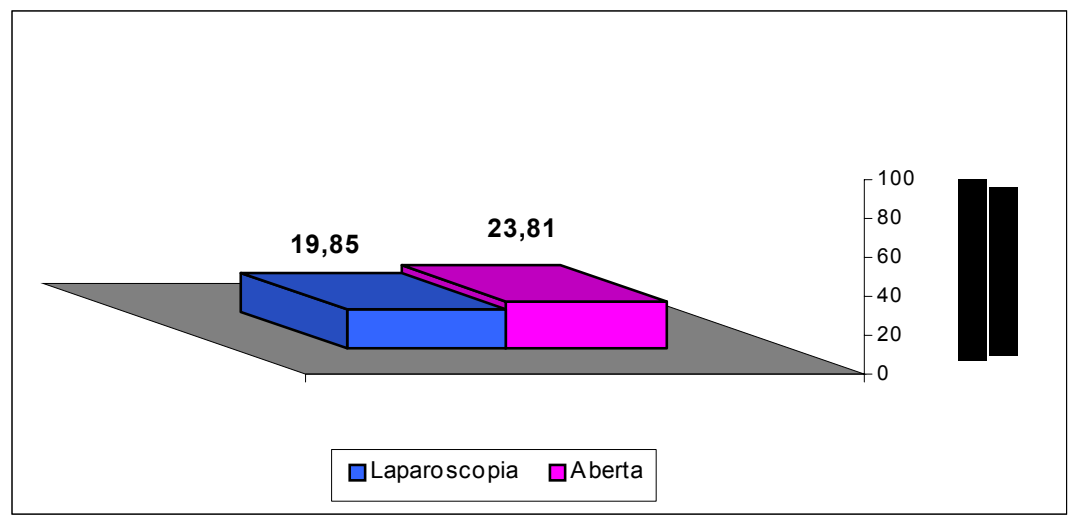

Figura 4. Percentual de observações de vocalização pós-operatória $(\mathrm{n}=210)$ nos dois grupos estudados $(\mathrm{P}=0,844-$ teste exato de Fisher $)$.

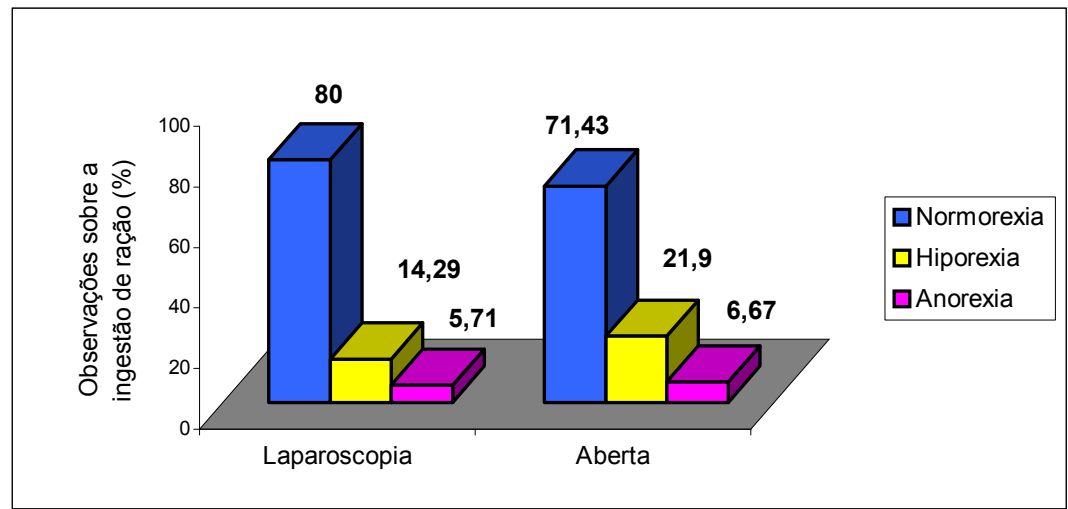

Figura 5. Percentual de observações sobre a ingesta pós-operatória, de acordo com o escore utilizado e o grupo estudado $(\mathrm{n}=210)\left(\mathrm{P}=0.321-\right.$ teste $\left.\chi^{2}\right)$.

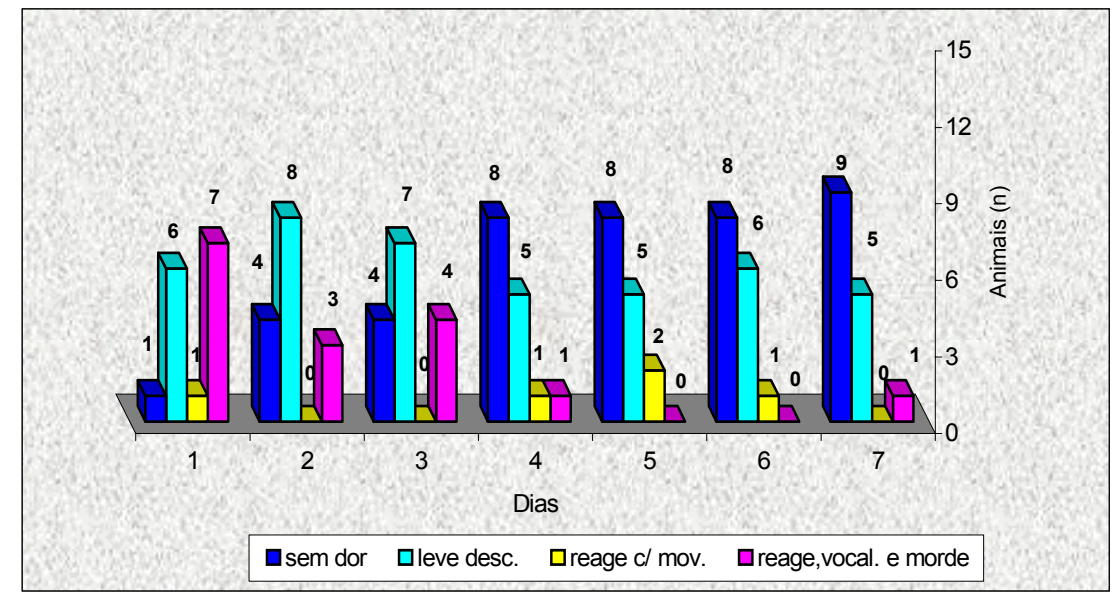

Figura 6. Dor ou desconforto dos animais do grupo I, segundo a avaliação por palpação do sítio cirúrgico, de acordo com o dia da avaliação ( $\mathrm{P}<0,05$ no dia 1 em relação aos dias 4, 5, 6, 7 - teste de KruskalWallis). 


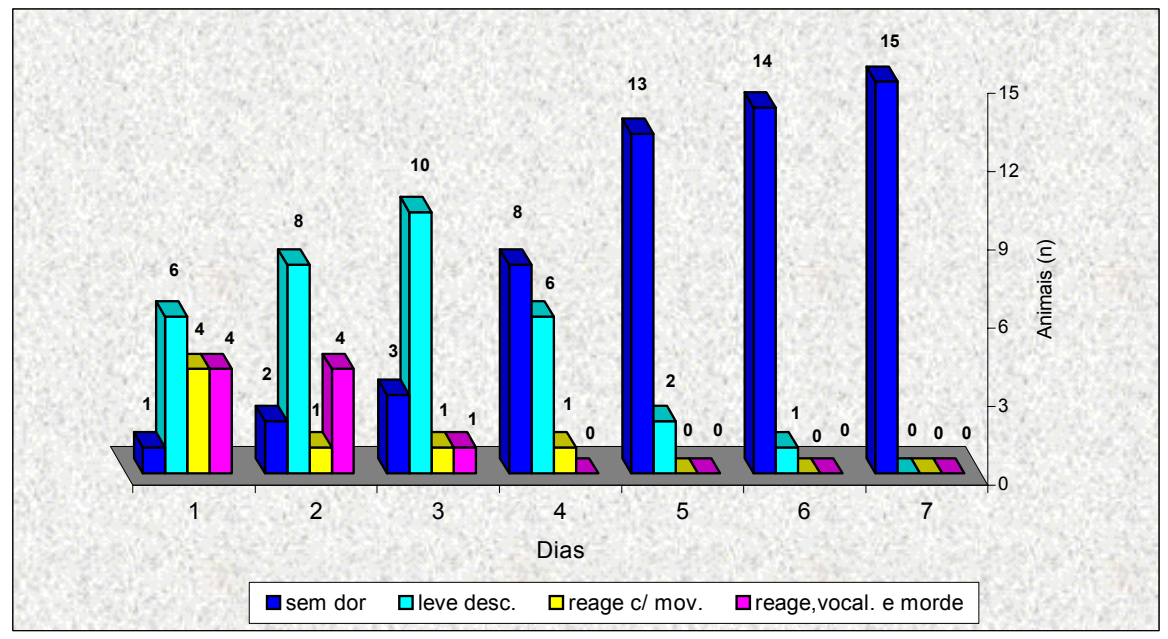

Figura 7. Manifestação de dor ou desconforto dos animais do grupo II segundo a avaliação por palpação do sítio cirúrgico, de acordo com o dia da avaliação ( $\mathrm{P}<0,05$ nos dias 1,2 e 3 em relação aos dias 5 , 6 e 7teste de Kruskal-Wallis).

Houve diferença significativa entre os grupos na resposta à palpação do sítio cirúrgico nos dias 5 $(\mathrm{P}=0,021), 6(\mathrm{P}=0,007)$ e $7(\mathrm{P}=0,004)$, com evidência de que as cadelas submetidas à abordagem aberta mostraram, à palpação da área operada, menor reação de desconforto e dor nesses dias (Fig. 6 e 7). Na Fig. 8 vê-se a evolução da dor ou desconforto, em ambos os grupos, com base nas médias ponderadas, considerando os quatro escores de reação à palpação da área operada, em cada dia de avaliação.

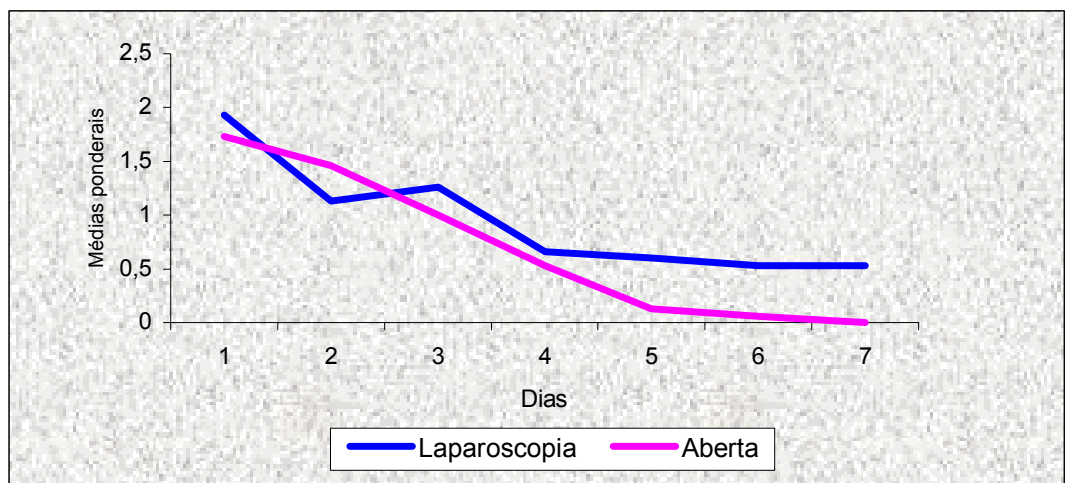

Figura 8. Evolução da dor ou desconforto à palpação do sítio cirúrgico, no pós-operatório e nos dois grupos estudados. Os valores das médias ponderadas correspondem à intensidade da dor.

No grupo I, no transcorrer dos sete dias do pósoperatório, ocorreram quatro casos de enfisema subcutâneo, um de hematoma, seis de seroma, cinco de deiscência dos pontos de pele e duas ocorrências de infecção nos portais. No grupo II, ocorreu um caso de seroma na ferida cirúrgica.

Ao longo de sete dias, os valores do escore 1 foram diferentes entre os grupos apenas no segundo dia do pós-operatório $(\mathrm{P}=0,034)$, quando a dor foi mais evidente nas cadelas submetidas à cirurgia aberta (Fig. 9). A dor manifestada pelos animais, na escala proposta (zero a 19), pôde ser classificada como de intensidade leve. $\mathrm{O}$ escore 2 evidenciou maior ocorrência de complicações pós-operatórias nas cadelas do grupo I (Fig. 10). A Fig. 11 representa o escore total e evidencia que a recuperação pósoperatória foi semelhante entre as duas abordagens. 


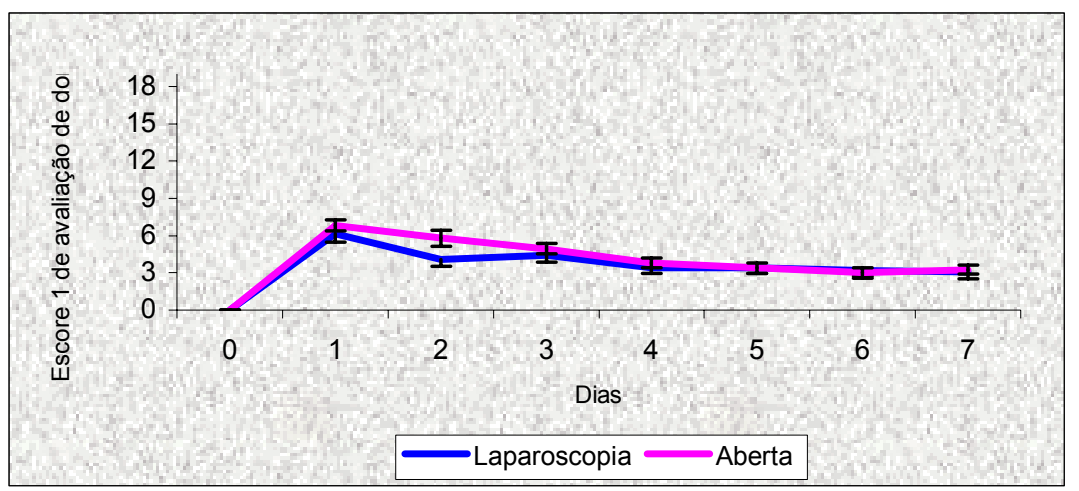

Figura 9. Evolução da dor ou desconforto no pós-operatório nos dois grupos estudados considerando-se os escores 1 médios $(\mathrm{I}=$ epm). Observou-se diferença entre os grupos. $(\mathrm{P}=0,03417)$ apenas no dia 2 (teste de Wilcoxon).

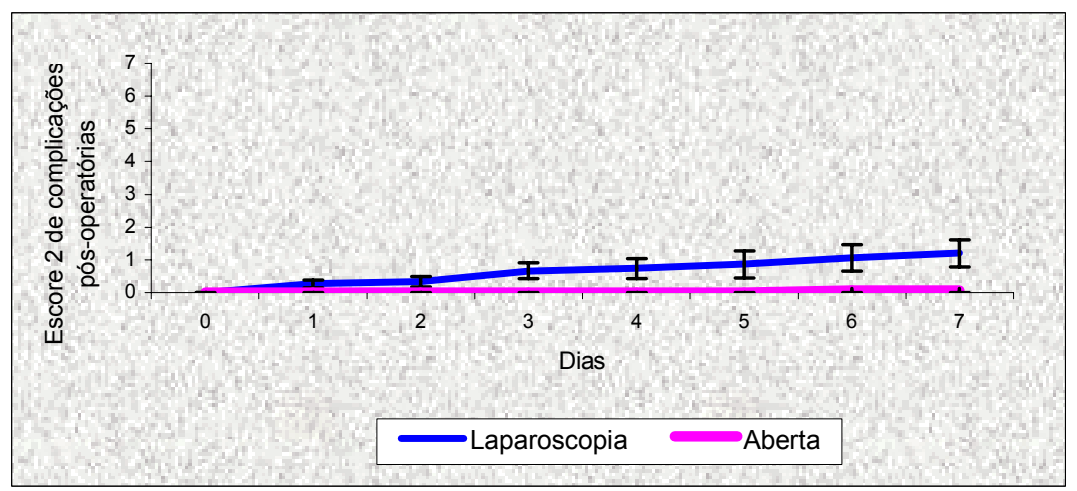

Gráfico 10. Complicações nas feridas cirúrgicas nos dois grupos estudados considerando-se os escores 2 médios $(\mathrm{I}=\mathrm{epm})$. Observou-se diferença entre os grupos nos dias $1-\mathrm{P}=0,01733 ; 2-\mathrm{P}=0,01751 ; 3-\mathrm{P}=$ 0,$00375 ; 4-\mathrm{P}=0,00375 ; 5-\mathrm{P}=0,00375 ; 6-\mathrm{P}=0,00238$ e 7-P =0,00098 (teste de Wilcoxon).

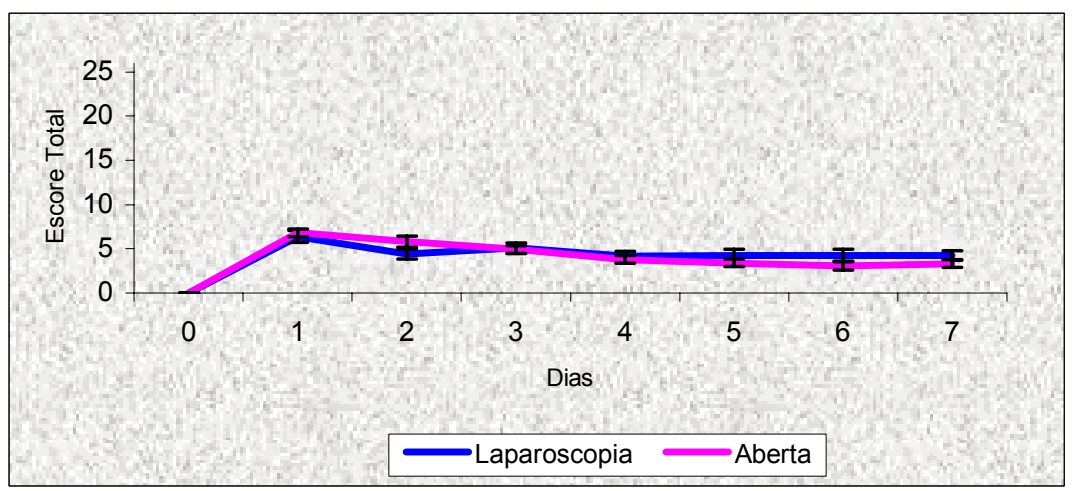

Figura 11. Evolução clínica pós-operatória nos dois grupos estudados considerando-se o escore total (escore $1+$ escore 2$)(\mathrm{I}=\mathrm{epm})$. Não foram observadas diferenças entre os grupos ao longo dos sete dias de pós-operatório (teste de Wilcoxon - $\mathrm{P}>0,05$ ). 


\section{DISCUSSÃO}

$\mathrm{Na}$ medicina veterinária, a avaliação da dor baseia-se na observação e na interpretação subjetiva de padrões de comportamento complexos que variam em função da espécie, raça, sexo, idade, condicionamento prévio, condições do meio ambiente, dominância social, saúde e individualidade (Johnson, 1991; Hardie et al., 1997; Hardie, 2002). Parâmetros fisiológicos, mesmo sendo inespecíficos, são também utilizados em escalas multidimensionais, juntamente com alterações do comportamento, no intuito de avaliar e mensurar a dor nos animais (Sackman, 1991; Hellyer e Gaynor, 1998; Firth e Haldane, 1999). Na medicina humana, estimar a intensidade da dor também é extremamente difícil, e variáveis fisiológicas do comportamento bem como relatos do paciente são usados com esse intuito (Tyler et al., 1993; Merkel e Malviya, 2000).

A dor é uma experiência para a qual não há medida direta, no entanto, com o melhor entendimento sobre a nocicepção, descobriu-se que números podem ser aplicados a certos fenômenos comportamentais e fisiológicos, atribuindo-lhes mais respeitabilidade e segurança. Dessa forma, vários sistemas de escore e escalas têm sido utilizados com a finalidade de avaliar e mensurar a dor (Wall, 1992; Mathews, 2000; Hardie, 2002).

Neste estudo, mediante a utilização de uma escala com avaliação de parâmetros clínicos e fisiológicos, foi possível comparar o desconforto e a dor pós-operatória entre as duas abordagens cirúrgicas estudadas. Em resposta à dor, os animais podem exibir sinais, como indiferença ao ambiente e às pessoas, ausência de movimentação, redução das atividades, redução ou ausência do apetite, enquanto outros apresentam inquietação, agressividade e tentativas de fuga (Hansen, 1997; Hellyer e Gaynor, 1998; Mathews, 2000; Hardie, 2002). Postura de cifose, sentar ou deitar em posição anormal, aumento do tônus da musculatura abdominal e interferência na área operada foram também relatados por esses autores. No presente estudo, as cadelas, em ambos os grupos, apresentaram alterações na postura corporal, no apetite, na vocalização, na tensão abdominal à palpação, além de interferência na área operada e comportamento de tranqüilidade ou agressividade. Os sinais permitiram classificar a dor de acordo com o escore proposto. $\mathrm{O}$ fato de não ter ocorrido diferença significativa entre os dois grupos quanto às variáveis citadas pode estar relacionado com o tipo de cirurgia. A OVH é um procedimento que determina dor pósoperatória de intensidade leve a moderada, que varia em função da duração e da extensão do procedimento, do grau de manipulação dos tecidos, da idade e do escore corporal do animal (Firth e Haldane, 1999; Mathews, 2000) A classificação de procedimentos cirúrgicos, estados mórbidos e traumas que determinam a sensação dolorosa dependem do tipo de estímulo nociceptivo inicial (Johnson, 1991; Mathews, 2000).

Enfisema subcutâneo, observado nas cadelas do grupo I, tem sido relatado em humanos (Litwin et al., 1992; Savassi-Rocha, 1995), em eqüinos (Bouré et al., 1997) e em cães (Brun, 1999) e, na maioria dos casos, o problema se resolve espontaneamente pela reabsorção do gás, sem maiores conseqüências danosas para o paciente (Coelho, et al., 1995; De Lisle et al., 1995). Outras ocorrências, como hematoma, seroma, infecção e deiscência, verificadas no presente estudo, são relatadas nas cirurgias laparoscópicas em humanos (Schirmer et al., 1991; Soper e Dunegan, 1993; Savassi-Rocha, 1995, SavassiRocha et al., 1997), no entanto são citadas como sendo mais freqüentes na técnica convencional. As infecções ocorrem mais comumente nos locais de introdução de trocartes, principalmente no umbigo, local de retirada de estruturas abdominais e de acúmulo de secreções (Coelho et al., 1995).

As complicações ocorridas nas feridas cirúrgicas foram mais observadas nas cadelas submetidas à laparoscopia, o que difere dos relatos na medicina humana. Essas alterações parietais foram observadas em todos os portais e não apenas naquele da retirada de vísceras, como previamente citado em humanos. Além disso, neste estudo, o portal para retirada de vísceras situou-se na região retroumbilical paramediana direita, entre a quarta e quinta glândulas mamárias (Malm et al., 2004). Alguns fatores, como: trauma local por excessiva e demorada manipulação das cânulas e compressão nas bordas das feridas, falhas na hemostasia de pequenos vasos subcutâneos, comprometimento dos linfáticos próximos às glândulas mamárias e 
possíveis resíduos de glutaraldeído no instrumental laparoscópico podem explicar a ocorrência das complicações parietais. Swaim (1980) e Waldron e Trevor (1996) consideram que a técnica cirúrgica traumática e a transeção de tecidos ricos em vasos linfáticos, dentre outros fatores, estão diretamente associados ao desenvolvimento de seroma que, eventualmente, evolui para infecção da ferida cirúrgica. As complicações parietais podem ter contribuído para maior desconforto ou maior manifestação da dor nas cadelas do grupo I, por ocasião da palpação da área operada. Segundo Pascoe (1996), os tecidos lesados liberam mediadores químicos que afetam a circulação local e estimulam os nociceptores da área. Nessas condições, a sensibilidade ao estímulo mecânico está aumentada tanto no sítio cirúrgico como nos tecidos adjacentes.

Na avaliação do escore 1, houve manifestação mais intensa de dor no primeiro dia do pósoperatório, independente do tipo de abordagem cirúrgica, que diminuiu gradativamente até o sétimo dia. Estes resultados estão de acordo com Hansen (1997), ao comentar que a dor pósoperatória apresenta curso previsto, com pico entre 6 e 24 horas após a cirurgia, que diminui progressivamente. Quando as duas abordagens foram comparadas, os valores do escore 2 mostraram que houve maior ocorrência de complicações nas feridas cirúrgicas na cirurgia laparoscópica. Savassi-Rocha (1995), Cooperman, (1995) e Malafaia e Campos (1995) comentaram que, na medicina humana, as complicações parietais são de baixa incidência, pois as infecções nos locais de inserção de trocartes são raras e facilmente tratadas. Apesar das diferenças observadas entre os grupos nos escores 1 e 2, quando estes foram agrupados (escore total), ficou evidente que a recuperação pós-operatória foi semelhante entre os dois grupos.

\section{CONCLUSÃO}

Pode-se concluir que a evolução clínica e a recuperação pós-operatória das cadelas submetidas à ovário-histerectomia pelas abordagens laparoscópica e aberta se equivalem, embora a incidência de complicações nas feridas cirúrgicas seja maior nas cadelas operadas pela laparoscopia.

\section{REFERÊNCIAS BIBLIOGRÁFICAS}

AGUILAR, R.F.; MIKOTA, S.K.; SMITH, J. Endoscopic ovariohysterectomy in two lions (panthera leo). J. Zoo Wildlife Med., v.28, p.290-297, 1997.

ANDREOLLO, N.A.; COELHO NETO, J.S.; LOPES, L.R.A. laparoscopia no diagnóstico das doenças intraabdominais. Análise de 168 casos. Rev. Assoc. Med. Bras., v.45, p.34-38, 1999.

BOURÉ, L.; MARCOUX, M.; LAVERTY, S. Paralumbar fossa laparoscopic ovariectomy in horses with use of endoloop ligatures. Vet. Surg., v.26, p.478483, 1997.

BRUN, M.V. Ovário-histerectomia em caninos por cirurgia laparoscópica. 1999. 181 f. Dissertação (Mestrado em Ciências Veterinárias) - Faculdade de Veterinária, Universidade Federal do Rio Grande do Sul, Porto Alegre.

COELHO, J.C.U.; MARCHESINI, J.B.; WIEDERKEHR, J.C. Complicações gerais em videocirurgia. In: COELHO, J.C.U.; MARCHESINI, J.B.; MALAFAIA, O. Complicações da videocirurgia - da profilaxia ao tratamento. Rio de Janeiro: MEDSI, 1995. cap. 4, p. 27-47.

COOPERMAN, A.M. Complications of laparoscopic surgery: In: ARREGUI, M.E.; FITZGIBBONS, R.J.; KATKHOUDA, N. et al. Principles of laparoscopic surgery: basic and advances techniques. New York: Springer-Verlag, 1995. cap. 7, 71-77.

DE LISLE, N.P.; JACKSON, K.D.; PASCHALL, V. et al. The team: nursing's perspective. In: ARREGUI, M.E.; FITZGIBBONS, R.J.; KATKHOUDA, N. et al. Principles of laparoscopic surgery: basic and advances techniques. New York: Springer-Verlag, 1995. cap. 9, p.91-100.

FIRTH, A.M.; HALDANE, S.L. Development of a scale to evaluate postoperative pain in dogs. J. Am. Vet. Med. Assoc., v. 214, p.651-659, 1999.

FRAZZE, M.D.; ROBERTS, J.W.; OKESON, G.C. et al. Open versus laparoscopic cholecystectomy - A comparison of postoperative pulmonary function. Ann. Surg., v.213, p.651-653, 1991.

GRAVES, H.A.; BALLINGER, J.F.; ANDERSON, W.J. Appraisal of laparoscopic cholecystectomy. Ann. Surg., v.213, p.655-664, 1991.

HANSEN, B. Through a glass darkly: using behaviour to assess pain. Semin. Vet. Med. Surg.: Small Anim., v.12, p.61-74, 1997.

HANSEN, B.D.; HARDIE, E.M.; CARROL, G.S. Physiological measurements after ovariohysterectomy in dogs: what's normal? Appl. Anim. Behav. Sci., v.51, p.101-109, 1997. 
HARDIE, E.M. Reconhecimento do comportamento doloroso em animais. In: HELLEBREKERS, L. J. Dor em animais: uma abordagem com orientação prática para um controle eficaz da dor em animais. 1.ed. São Paulo: Manole, 2002. cap. 4, p.49-68.

HARDIE, E.M.; HANSEN, B.D.; CARROLL, G.S. Behavior after ovariohysterectomy in the dog: what's normal? Appl. Anim. Behav. Sci., v.51, p.111-128, 1997.

HELLYER, P.W.; GAYNOR, J.S. Acute postsurgical pain in dogs and cats. Comp. Small Anim., v.20, p.140153, 1998.

HENDOLIN, H.I.; PÄÄKKÖNEN, M.E.; ALHAVA, E.M. et al. Laparoscopic or open cholecystectomy: A prospective randomised trial to compare postoperative pain, pulmonary function and stress response. Europ. J. Surg., v.166, p.394-399, 2000.

JOHNSON, J.M. The veterinarian's responsability assessing and managing acute pain in dogs and cats. Part I. Comp. Small Anim., v.13, p.804-807, 1991.

LITWIN, D.E.M.; GIROTTI, M.J.; POULIN, E.C. Laparoscopic cholecystectomy: trans-Canada experience with 2201 cases. Can. J. Surg., v.35, p.291-296, 1992.

MALAFAIA, O.; CAMPOS, A.C.L. Infecção em videocirurgia In: COELHO, J.C.U.; MARCHESINI, J.B.; MALAFAIA, O. Complicações da videocirurgia: da profilaxia ao tratamento. Rio de Janeiro: MEDSI, 1995. cap. 5, p.49-55.

MALM, C.; SAVASSI-ROCHA, P.R.; GHELLER, V.A. et al. Ovário-histerectomia: estudo experimental comparativo entre as abordagens laparoscópica e aberta na espécie canina. Intra-operatório-I. Arq. Bras. Med. Vet. Zootec., v.56, p.457-466, 2004.

MATHEWS, K.A. Pain assessment and general approach to management. Vet. Clin. North Am.: Small Anim. Pract., v.30, p.729-755, 2000.

MERKEL, S.; MALVIYA, S. Pediatric pain, tools and assessment. J. Peri Anesth. Nurs., v.15, p.408-414, 2000 .

MINAMI, S.; OKAMOTO, Y.; EGUCHI, H. et al. Successful laparoscopy assisted ovariohysterectomy in two dogs with pyometra. J. Vet. Med. Sci., v.59, p.845-847, 1997.
PASCOE, P.J. Patient aftercare In: SLATTER, D. Textbook of small animal surgery. 2.ed. Philadelphia: W.B. Saunders, 1996. cap.22, p.230-240.

ROLLIN, B.E. A ética do controle da dor em animais de companhia. In: HELLEBREKERS, L.J. Dor em animais: uma abordagem com orientação prática para um controle eficaz da dor em animais. 1.ed. São Paulo: Manole, 2002. cap.2, p.17-35.

SACKMAN, J.E. Pain: its perception and alleviation in dogs and cats. Part I. The physiology of pain. Comp. Small Anim., v.13, p.71-75, 1991.

SAVASSI-ROCHA, P.R. Colecistectomia. In: COELHO, J.C.U.; MARCHESINI, J.B.; MALAFAIA, O. Complicações da videocirurgia: da profilaxia ao tratamento. Rio de Janeiro: MEDSI, 1995. cap.13, p.183-241.

SAVASSI-ROCHA, P.R.; FERREIRA, J.T.; COSTA DINIZ, M.A. Laparoscopic cholecystectomy in Brazil: analysis of 33.563 cases. Int. Surg., v.82, p.208-213, 1997.

SCHIRMER, B.D.; EDGE, S.B.; DIX, J. et al. Laparoscopic cholecystectomy. Treatment of choice for symptomatic cholelithiasis. Ann. Surg., v.213, p.665-677, 1991.

SOPER, N.J.; DUNNEGAN, D.L. Laparoscopic cholecystectomy: experience of a single surgeon. World J. Surg., v.17, p.16-21, 1993.

SWAIM, S.F. Management of contaminated and infected wounds. In: SWAIM, S.F. (Ed.). Surgery of traumatized skin: management and reconstruction in the dog and cat. Philadelphia: W.B. Saunders, 1980. cap.4, p.119-213.

TYLER, D.C.; JOANNE DOUTHIT, A.T.; CHAPMAN, C.R. Toward validation of pain measurement tools for children: a pilot study. Pain, v.52, p.301-309, 1993.

WALDRON, D.R.; TREVOR, P. Management of superficial skin wounds. In: SLATTER, D. Textbook of small animal surgery. 2.ed. Philadelphia: W.B. Saunders, 1996. cap.25, p.269-280.

WALL, P.D. Defining pain in animals. In: SHORT, C.E.; POZNbAK, A.V. (Eds.). Animal Pain. New York: Churchill Livingstone, 1992. cap.3, p.63-79. 